$1-3-2006$

\title{
Equation of State and Beginning of Thermalization After Preheating
}

Dmitry Podolsky

University of Toronto

Gary Felder

Smith College, gfelder@smith.edu

Lev Kofman

University of Toronto

Marco Peloso

University of Minnesota - Twin Cities

Follow this and additional works at: https://scholarworks.smith.edu/phy_facpubs

Part of the Physics Commons

\section{Recommended Citation}

Podolsky, Dmitry; Felder, Gary; Kofman, Lev; and Peloso, Marco, "Equation of State and Beginning of Thermalization After Preheating" (2006). Physics: Faculty Publications. 11.

https://scholarworks.smith.edu/phy_facpubs/11 


\title{
Equation of state and beginning of thermalization after preheating
}

\author{
Dmitry Podolsky, ${ }^{1, *}$ Gary N. Felder, ${ }^{2}$ Lev Kofman, ${ }^{1}$ and Marco Peloso ${ }^{3}$ \\ ${ }^{1}$ CITA, University of Toronto, 60 St. George Street, Toronto, Ontario M5S 3H8, Canada \\ ${ }^{2}$ Department of Physics, Clark Science Center, Smith College, Northampton, Massachusetts 01063, USA \\ ${ }^{3}$ School of Physics and Astronomy, University of Minnesota, Minneapolis, Minnesota 55455, USA
}

(Received 8 July 2005; published 3 January 2006)

\begin{abstract}
We study the out-of-equilibrium nonlinear dynamics of fields after post-inflationary preheating. During preheating, the energy in the homogeneous inflaton is exponentially rapidly transfered into highly occupied out-of-equilibrium inhomogeneous modes, which subsequently evolve towards equilibrium. The infrared modes excited during preheating evolve towards a saturated distribution long before thermalization completes. We compute the equation of state during and immediately after preheating. It rapidly evolves towards radiation domination long before the actual thermal equilibrium is established. The exact time of this transition is a nonmonotonic function of the coupling between the inflaton and the decay products, and it varies only very weakly (around $10^{-35} \mathrm{~s}$ ) as this coupling changes over several orders of magnitude. This result is applied to refine the relation between the number of e-foldings $N$ and the physical wavelength of perturbations generated during inflation. We also discuss the implications for the theory of modulated perturbations from preheating. We finally argue that many questions of the thermal history of the universe should be addressed in terms of prethermalization, illustrating this point with a calculation of perturbative production of gravitinos immediately after chaotic inflation. We also highlight the effects of three-legs inflaton interactions on the dynamics of preheating and thermalization in an expanding universe.
\end{abstract}

DOI: 10.1103/PhysRevD.73.023501

PACS numbers: $98.80 . \mathrm{Cq}, 11.10 .-\mathrm{z}$

\section{INTRODUCTION: BETWEEN INFLATION AND THERMALIZATION}

According to the inflationary scenario, the universe at early times expands quasiexponentially in a vacuumlike state without entropy or particles. During this stage of inflation, all energy is contained in a classical slowly moving inflaton field $\phi$. The fundamental Lagrangian $\mathcal{L}\left(\phi, \chi, \psi, A_{i}, h_{i k}, \ldots\right)$ contains the inflaton part with the potential $V(\phi)$ and other fields which give subdominant contributions to gravity. In chaotic inflationary models, soon after the end of inflation the almost homogeneous inflaton field $\phi(t)$ coherently oscillates with a very large amplitude of the order of the Planck mass around the minimum of its potential. Because of the interactions of other fields with the inflaton in $\mathcal{L}$, the inflaton field decays and transfers all of its energy to relativistic particles. If the creation of particles is sufficiently slow (for instance, if the inflaton is coupled only gravitationally to the matter fields) the decay products simultaneously interact with each other and come to a state of thermal equilibrium at the reheating temperature $T_{r}$. This gradual reheating can be treated with the perturbative theory of particle creation and thermalization as long as the couplings are sufficiently small [1-3]. However, the particle production from a coherently oscillating inflaton for a wide range of couplings occurs in a nonperturbative regime of parametric excitation [4-6]. This picture, with variation in its details, is extended to

\footnotetext{
*On leave from Landau Institute for Theoretical Physics, 117940, Moscow, Russia
}

other inflationary models. For instance, in hybrid inflation inflaton decay proceeds via a tachyonic instability of the inhomogeneous modes which accompany the symmetry breaking [7].

One consistent feature of preheating - nonperturbative copious particle production immediately after inflationis that the process occurs far away from thermal equilibrium. The energy of the inflaton zero mode is transferred to particles in an out-of-equilibrium state with very large occupation numbers within a very short time interval of about $10^{-35} \mathrm{sec}$, which can be much shorter than the time needed for relaxation towards thermal equilibrium.

One can expect the initial conditions for many parameters of the subsequent cosmological thermal history to be settled during or around preheating. It is often thought that the details of the transition between inflation and the hot radiation dominant stage are not relevant, except for the socalled reheating temperature $T_{r}$, the temperature of the ultrarelativistic plasma at the time when it reaches thermal equilibrium. Definitely, this is an important parameter of the early universe. However, a precise understanding of how thermal equilibrium is reached is crucial since partial thermal distributions can be responsible for cosmological baryo/leptogenesis, the possible creation of dangerous cosmological relics, etc. The out-of-equilibrium character of preheating opens the possibility for equally relevant phenomena associated with nonequilibrium physics, including phase transitions, nonthermal production of heavy particles, etc.

Precise knowledge of the expansion of the universe $a(t)$ is also required to connect a physical wavelength of the 
observed cosmological perturbation, $k / a(t)$, to the number of e-foldings $N$ at which that wavelength exited the horizon during inflation.

Finally, we can have an alternative mechanism of generation of (almost) scale free adiabatic metric perturbations from preheating, based on spatial modulation of couplings [8-10]. Small spatial fluctuations in the couplings lead to small fluctuations of the rates of physical processes, which in turn generate adiabatic perturbations of the energy density and of the metric. Analyzing the details of this mechanism require precise knowledge of the evolution of the equation of state (EOS).

To understand the early post-inflationary period we have to understand the dynamics of interacting fields in an outof-equilibrium state with large occupation numbers evolving towards a state of ultimate thermal equilibrium. This is a complicated problem of nonequilibrium quantum field theory, which is by itself a very interesting topic.

The theory of the transition from inflation to thermalization has been investigated with fully nonlinear numerical lattice simulations plus different techniques of classical field dynamics [11-15]. Classical field dynamics is adequate as long as occupation numbers of field excitations are large. There has also been progress in understanding outof-equilibrium QFT dynamics in $O(N)$ sigma models beyond the Hartree approximation [16], taking into account crucial effects of rescattering after preheating [17]. From all of these methods, the following picture emerges. Immediately after preheating, either from parametric resonance or tachyonic, one or more bose fields are excited in an out-of-equilibrium state with large occupation number. Backreaction of these fields terminates their production. Interaction (rescattering) of these modes between each other, and with the remaining inflaton field after the first stage of preheating is violent and nonperturbative. During this very short stage a large amount of entropy is generated, chaotic (turbulent) wave dynamics is established, and the fields not directly excited during preheating get excited in out-of-equilibrium states due to rescattering; residual inflaton oscillations are still present. A next, longer stage then takes place, characterized by lower (but still large) occupation numbers such that rescattering is perturbative, and the occupation spectra gradually move towards a saturated state by cascading towards ultraviolet (UV) and infrared (IR) modes (in the spirit of Kolmogorov wave turbulence). The last and the longest stage will be the stage of proper thermalization, when the distributions evolve towards thermal equilibrium. Quantum physics is important at the end of this stage, when the occupation numbers are small and the classical approximation breaks down. One may say that reheating is completed when this last, longest stage is completed, and its timing defines $T_{r}$ and $a_{r}$.

In this paper we suggest a new look at the transition between inflation and thermalization. We focus on calculation and understanding of the effective equation of state $w=p / \epsilon$ throughout all of these stages after inflation. We use numerical lattice simulations [15] to calculate $w$ in the simple chaotic model with a quadratic potential and a simple four-legs interaction $g^{2} \phi^{2} \chi^{2}$.

In particular, we notice that $w$ approaches (but does not necessarily reach) the equilibrium radiation-dominated value $1 / 3$ while the fields are far from ultimate thermal equilibrium. We compare this with the analysis of [18], devoted to thermalization of the $\mathrm{O}(N)$ sigma model in Minkowski spacetime. In that case, the equation of state was found to evolve sharply towards $w=1 / 3$ long before thermalization completes, which prompted the authors of [18] to describe the state of the system as a prethermalized state. Although we also see a somewhat similar effect, the expansion of the universe and the presence of a residual massive inflaton component, prevent the equation of state from being exactly the one of radiation at this very early stage. Moreover, we emphasize that the exact microphysics of the system is described by a turbulent state, which is still very far from a thermalized one. (This difference can be important when discussing the cosmological effects of decay products, as for instance production of dangerous relics from nonthermal distributions.) Indeed, our simulations show that the occupation numbers of excited infrared modes evolve towards a steady state, related to turbulence of classical interacting waves.

The plan of the paper is the following: In Sec. II we describe preheating in a simple chaotic inflationary model. In Sec. III we present the results of lattice simulations for time-dependent variables, including the equation of state $w(t)$ during different stages of preheating, occupation numbers and fluctuations.

In Sec. IV we discuss some immediate applications of our results for cosmology. We consider the application of the EOS evolution to modulated cosmological fluctuations and to the $N-\log k$ formula. We note several qualitatively important issues in the dynamics of thermalization. The interaction $\phi^{2} \chi^{2}$ describes (at the perturbative level) scattering between inflaton quanta, rather than inflaton decay. Scattering between massive $\phi$ soon becomes inefficient, and does not lead to a complete depletion of the inflaton, which eventually ends up dominating over the light degrees of freedom $\chi$. This problem is automatically avoided if three-legs bosonic interactions $\phi \chi^{2}$ are also present. We illustrate this with an example motivated by supersymmetry. We also discuss the rapid saturation of the IR modes excited during preheating, which occurs long before the ultimate thermal equilibrium (of all modes) is reached.

One consequence of the excitation of infrared modes is that it can lead to an overproduction of gravitinos or of other dangerous gravitational relics. This generation is different from direct nonperturbative production during preheating [19]. Instead, it is analogous to the perturbative thermal production first considered in [20]. The main difference with [20] is that we compute the production 
right after the first preheating stage, and not only after thermalization has completed [20]. Although the distributions formed at preheating/rescattering are far from thermal, the high particle occupation numbers can lead to a significant generation of gravitinos.

The above results are summarized in Sec. V.

\section{THE MODELS AND METHODS}

In this section we briefly describe the model of chaotic inflation with a quadratic potential and additional interaction terms and the methods we use to investigate the fields dynamics. The quadratic form of the potential is a good generic approximation around the minimum. For simplicity, we take the potential

$$
V(\phi)=\frac{1}{2} m^{2} \phi^{2},
$$

also throughout inflation. The inflaton mass is taken to be $m \simeq 10^{13} \mathrm{GeV}$, which, in the simplest cases, reproduces the correct amplitude for the CMB anisotropies. After inflation, the background inflaton field oscillates with its amplitude diluted by the expansion as $\phi \propto \phi_{0} / a^{3 / 2}$, where $\phi_{0} \approx M_{p} / 10$ is the initial amplitude, and $a(t)$ is the scale factor of the FRW flat universe. More accurately, the inflaton evolution is given by $\phi(t) \approx\left(M_{P} / \sqrt{3 \pi} m t\right) \times$ $\sin m t$. The energy density of the inflaton oscillations evolves as $\epsilon \approx m^{2} \phi_{0}^{2} / a^{3}$, while the pressure is $p \approx$ $-\left(m^{2} \phi_{0}^{2} / a^{3}\right) \cos 2 m t$. Since the frequency of the oscillations is higher than the rate of expansion $H$, we deal with a nearly vanishing averaged effective pressure, $p \approx 0$. Therefore, immediately after inflation, the equation of state (EOS) is effectively the one of matter domination

$$
w=p / \epsilon \approx 0 .
$$

This is what we expect for inflaton oscillations interpreted as the coherent superposition of heavy inflaton (quasi)particles at rest.

Inflaton oscillations decay due to the coupling between the inflaton and other fields. One may think about the inflaton coupling to other bosons through four-legs or three-legs interactions, for instance coupling to another scalar $\chi$ as $g^{2} \phi^{2} \chi^{2}$ or $g^{2} \sigma \phi \chi^{2}$. Yukawa-type couplings to fermions will be of the form $h \bar{\psi} \phi \psi$. During preheating the leading channel of inflaton decay will be parametric resonance decay into bosons. A three-legs interaction between bosons $g^{2} \sigma \phi \chi^{2}$ requires a dimensionful scale $\sigma$, which emerges naturally in SUSY theories. However, in most cases the amplitude of the inflaton is sufficiently high during the early stages of preheating that the four-legs interaction dominates [21]. For this reason, at this stage we consider only the four-legs interaction

$$
\mathcal{L}_{\text {int }}=-\frac{g^{2}}{2} \phi^{2} \chi^{2}
$$

Three-legs interactions are important for the completion of the decay of massive inflatons, and we will discuss them further in Sec. IV, and in detail in [21].

Before proceeding with the generation of $\chi$ particles from the inflaton oscillations, we need to comment on the range of the coupling $g^{2}$. At the level of QFT we can obtain an upper limit for $g^{2}$. Indeed, in non supersymmetric models, the interaction (3) leads to radiative corrections ${ }^{1}$ to the effective potential (1), of the form $\left(g^{4} \phi^{4} / 32 \pi^{2}\right) \ln \phi$. For inflaton values $\phi \sim 4 M_{p}$, which correspond to the scales where cosmological fluctuations are observed, radiative corrections do not alter the the potential (1) as long as $g^{2} \lesssim 10^{-5}$. In supersymmetric theories radiative corrections from bosons and fermions cancel each other out so that the value of $g$ can be even higher.

There is also a lower limit on $g^{2}$. During inflation the field $\chi$ has the effective mass $m_{\chi}^{2}+g^{2}\left\langle\phi^{2}\right\rangle \sim g^{2} M_{p}^{2}$, where $m_{\chi}$ is its "bare mass": we take $m_{\chi} \ll m$ and thus neglect it. It is known [22] that if we have two scalar fields $\phi$ and $\chi$, the latest stage of inflation will be driven by the lightest scalar (light scalars are always generated during inflation). A consistent setting where the end of inflation is driven by the $\phi$ field requires $g^{2} M_{p}^{2}>m^{2}$, i.e. $g^{2}>10^{-12}$. This lower limit can be evaded, however, if $\chi$ is conformally coupled to gravity.

A quantum scalar field $\chi$ in a flat FRW background has the eigenfunctions $\chi_{k}(t) e^{-i \vec{k} \vec{x}}$ with comoving momenta $\vec{k}$. The temporal part of the eigenfunction $\chi_{k}(t)=a^{3 / 2} X_{k}(t)$ obeys the equation

$$
\ddot{X}_{k}+\left(\frac{k^{2}}{a^{2}}+g^{2} \phi^{2}\right) X_{k}=0
$$

with positive-frequency initial conditions in the far past. In the effective frequency $\omega_{k}^{2} \approx\left(k^{2} / a^{2}\right)+g^{2} \phi^{2}$ we neglect small terms proportional to $H^{2}$. This is an oscillatorlike equation with a periodic frequency, which contains exponentially unstable solutions corresponding to parametric resonance. It is convenient to use a new time variable $z=$ $m t$. Then, the essential dimensionless coupling parameter which characterizes parametric amplification is $q=$ $\left(g^{2} \phi_{0}^{2}\right) / 4 m^{2}$. The limits on the coupling $g^{2}$ we have discussed above correspond to $10^{-3} \lesssim q \lesssim 10^{5}$. A large portion of this interval is in the broad parametric resonance regime $(q>1)$ of production of $\chi$ particles [4].

What is important, however, is that even one bosonic field with a large parameter $q$ is sufficient to have the leading channel of inflaton decay occur through broad parametric resonance. Indeed, if one such field $\chi$ is exponentially amplified in this way then other fields that are coupled to it directly or indirectly will tend to be amplified as well [13].

\footnotetext{
${ }^{1}$ There is also a divergent quadratic contribution. However, for the supersymmetric theory this term is independent of the value of $\phi$ and can thus be cancelled by a counter-term.
} 
We are interested in the dynamics of the fields $\phi$ and $\chi$ after inflation. Although this toy model is simple, its dynamics are rather rich and complex. The first stage is the stage of parametric excitation of the quantum fluctuations of the field $\chi$, where the light $\chi$ particles are copiously produced from the classical inflaton oscillation. This process is described by the theory of broad stochastic parametric resonance in an expanding universe [6].

As soon as the occupation number of created particles

$$
n_{k}=\frac{\omega_{k}}{2}\left(\frac{\left|\dot{X}_{k}\right|^{2}}{\omega_{k}^{2}}+\left|X_{k}\right|^{2}\right)-\frac{1}{2}
$$

becomes large, $n_{k} \gg 1$, one can approximate the dynamics with that of the classical interacting fields $\phi$ and $\chi$. Fully nonlinear wave equations for coupling classical fields are rather complicated. We use the LATTICEASY numerical simulations [15] to solve the equations $\square \phi+m^{2} \phi+$ $g^{2} \phi \chi^{2}=0$ and $\square \chi+g^{2} \phi^{2} \chi=0$ in an expanding universe ( $\square$ being the covariant wave operator in the FRW geometry). The scale factor $a(t)$ is self-consistently calculated from the Friedmann equation. The initial conditions correspond to Gaussian initial fluctuations of $\phi$ and $\chi$, which arise from vacuum fluctuations.

The novel element of our current study is the focus on new diagnostics of the numerical experiments. In particular, we investigate the evolution of the equation of state $w(t)$ during different stages, and for various couplings $g^{2}$. Many previous numerical studies of preheating concentrated on inflation with a quartic potential $V(\phi)=\lambda \phi^{4}$, where the expansion of the universe can be "scaled away". The inflaton oscillations in this model have the EOS $w=$ $1 / 3$, which corresponds to that of radiation domination, so $w$ does not change after the inflaton decay. By contrast, $w$ changes during preheating in the more general model with a quadratic potential.

The total energy density $\epsilon$ and pressure $p$ contain contributions from kinetic and gradient energies of the fields $\phi$ and $\chi$, plus interaction terms. It is instructive to calculate separate contributions from all of these terms. We find that the interaction term, except for a transient moment between the end of preheating and onset of turbulent regime, is sufficiently small that we can interpret the results in terms of different particles $\delta \phi$ and $\chi$ coupled to each other. In particular, this allows us to interpret the energy density in each component in terms of the integral

$$
\epsilon \approx \frac{1}{(2 \pi)^{3} a^{4}} \int d^{3} k \omega_{k} n_{k},
$$

where the comoving frequency is

$$
\omega_{k}=\sqrt{k^{2}+m_{\mathrm{eff}}^{2} a^{2}},
$$

and the comoving occupation number $n_{k}$ is given in (5). To study $w$ we have to monitor the relationship between the effective masses of the fields

$$
\begin{gathered}
m_{\phi, \text { eff }}^{2}=m^{2}+g^{2}\left\langle\chi^{2}\right\rangle, \\
m_{\chi, \text { eff }}^{2}=g^{2}\langle\phi\rangle^{2}+g^{2}\left\langle\delta \phi^{2}\right\rangle .
\end{gathered}
$$

and the typical momenta.

The variances of the fluctuations

$$
\begin{aligned}
\left\langle\chi^{2}\right\rangle & =\frac{1}{(2 \pi)^{3}} \int d^{3} k\left|\chi_{k}\right|^{2} \\
\left\langle\delta \phi^{2}\right\rangle & =\frac{1}{(2 \pi)^{3}} \int d^{3} k\left|\delta \phi_{k}\right|^{2},
\end{aligned}
$$

are also an important diagnostic of the nonequilibrium physics. Let us rewrite the wave function $X_{k}(t)$ in the WKB form $X_{k}(t)=\left(\alpha_{k} / \sqrt{2 \omega_{k}}\right) e^{-i \int d t \omega_{k}}+$ $\left(\beta_{k} / \sqrt{2 \omega_{k}}\right) e^{i \int d t \omega_{k}}$. While the occupation numbers in terms of $\alpha-\beta$ coefficients are simply $n_{k}=\left|\beta_{k}\right|^{2}$, the exact expression for the variances is more complicated

$$
\left\langle\chi^{2}\right\rangle=\frac{1}{(2 \pi)^{3} a^{3}} \int \frac{d^{3} k}{\omega_{k}}\left(n_{k}+2 \Gamma \operatorname{Re}\left(\alpha_{k} \beta_{k}^{*} e^{2 \int d t i \omega_{k}}\right)\right) .
$$

Particles $\chi$ produced at preheating are created in a squeezed state. In particular, this is reflected in the presence of the oscillatory second term in the integral (12), where the phases of $\alpha_{k}, \beta_{k}$ are present.

The total comoving number densities of $\chi$ and $\phi$ particles are

$$
\begin{aligned}
& N_{\chi}=\frac{1}{(2 \pi)^{3} a^{3}} \int d^{3} k n_{k}^{\chi}, \\
& N_{\phi}=\frac{1}{(2 \pi)^{3} a^{3}} \int d^{3} k n_{k}^{\phi},
\end{aligned}
$$

respectively. It is instructive to monitor the time evolution of the total number of particles $N_{\text {tot }}=N_{\chi}+N_{\phi}$. At leading order in $g^{2}$ the total number of particles is conserved, $N_{\text {tot }}(t)=$ const. However, there is a brief period immediately after preheating where $N_{\text {tot }}$ is poorly defined due to the strong interactions between the waves. Indeed, during preheating the occupation number of $\chi$ particles can be as large as $n_{k} \sim 1 / g^{2}$. Consider the collision integral in the kinetic equation for the particle. If we formally represent it as a series with respect to the coupling $g^{2}$, the smallness of $g^{2}$ will be compensated by the large occupation numbers. This means that the next terms of the collision integral associated with the higher order diagrams (for example, the diagram with four incoming, two outgoing legs, and two vertices) are as important as the first term. In fact, the nonperturbative character of the interactions during this short intermediate stage makes it difficult to investigate the whole dynamics analytically. After this period ends, the spectrum of the occupation numbers formed at preheating is weighted towards the infrared relative to a thermal 
distribution of the same energy density. Hence, particle fusion is more favored than particle dissociation. Once rescattering leads to a decrease of $n_{k}$, only the leading four-legs diagram with one vertex becomes important, and $N_{\text {tot }}$ decreases on the way to thermalization, on a timescale which is much longer than the one which can be observed in our lattice simulations.

\section{OUTPUT OF THE CALCULATIONS}

\section{A. The calculations}

We performed three-dimensional lattice simulations for the model of Sec. II. Our grid was a $256 \times 256 \times 256$ cube with a comoving edge size $L=10 / m$, which corresponds to a comoving grid spacing of $d x \approx 0.04 / \mathrm{m}$. As energy flows towards the UV end of the spectrum the simulations eventually reach a point where the grid spacing is too large to capture the important UV physics. By monitoring the spectra of the fields, however, we can verify that these simulation parameters were adequate to capture the relevant IR and UV physics well past the end of preheating. The time step was $d t=0.001 / \mathrm{m}$ and the inflaton mass was $m=10^{-6} M_{p}$. We used values of the coupling near $g^{2}=$ $10^{-7}$ This value is optimal because it is large enough to produce highly efficient preheating, but small enough that the occupation numbers $n_{k} \sim 1 / g^{2}$ produce strong rescattering. The results should be qualitatively similar for a wide range of values of $g^{2}$, but would require more IR and/or more UV to simulate accurately.

To probe later times and wider ranges of the couplings it will be necessary to extend the lattice simulations. This can be done with a parallelized version of the simulation code LATTICEEASY (currently under construction), or by combining the straightforward lattice simulations with other methods, like the equations for a large number of weakly coupled oscillators [23]. We intend to pursue both of these approaches in subsequent work.

In the rest of this section we present the results of our simulations.

\section{B. Equation of state}

The time evolution of the EOS $w(t)$ for different couplings is shown in Fig. 1. Each point plotted on this figure represents the value of $w$ averaged over a complete inflaton oscillation. This represents one of the main results of our study.

Immediately after inflation, the EOS averaged over inflaton oscillations is $w=0$. It sharply changes at the end of preheating.

There are at least three important points worth emphasizing about the evolution of $w$.

(i) First, the transition of the EOS from $w=0$ to the value $w \sim 0.2-0.3$ occurs very sharply, within a time interval $\sim 10^{-36} \mathrm{sec}$.

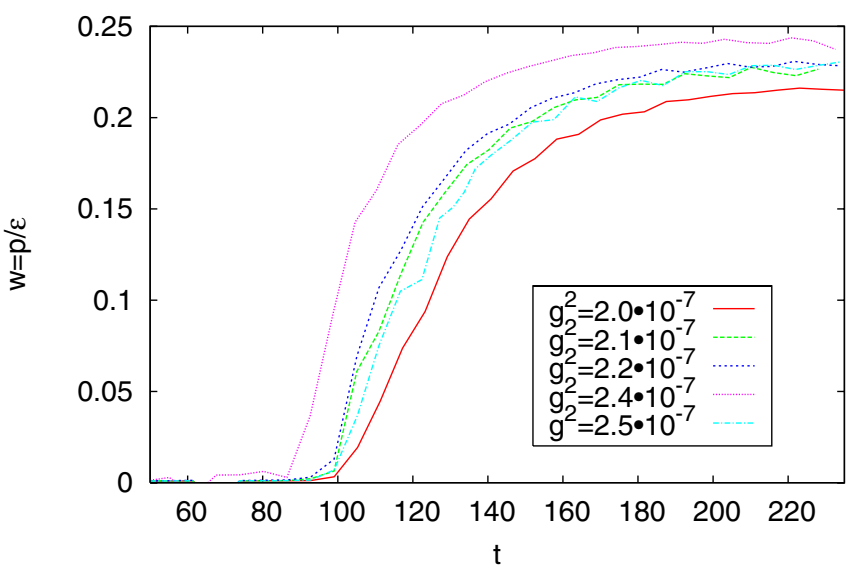

FIG. 1 (color online). Evolution of the equation of state $w=$ $w(t)$ as a function of time (given in units of $\mathrm{m}^{-1}$ ) for various couplings $g^{2}$ around $g^{2}=2 \times 10^{-7}$.

Indeed, recall that the unit of time on the plots is $1 / \mathrm{m}$, where $m$ is the inflaton mass, i.e. $10^{-37} \mathrm{sec}$. The first stage of preheating is completed within about a hundred of these units, i.e., $10^{-35} \mathrm{sec}$. The rise of $w$ and gradual saturation takes roughly the same time.

(ii) Second, the dependence of $w(t)$ on the coupling $g^{2}$ for resonant preheating is a nonmonotonic function of $g^{2}$.

This is to say that the time during which preheating comes to an end is very weakly (logarithmically) dependent on the coupling. As seen from Fig. 1 the curves $w(t)$ begin to shift to the left towards an earlier end of preheating, as we vary $g^{2}$ by $5 \%$. However, at some point the curves stop moving to the left and instead begin to return toward the right. As we change $g^{2}$ by about $25 \%$, the cycle repeats. As we vary $g^{2}$, the function $w$ not only shifts, but it also varies its detailed shape. Still, to characterize these variations, we pick up the moment where $w$ is equal to the value 0.15 (just for convenience of calculation), $w\left(t_{\text {tran }}\right)=$ 0.15 . This allows us to plot the transition moment $t_{\text {tran }}\left(g^{2}\right)$ as a function of $g^{2}$, see Fig. 2 .

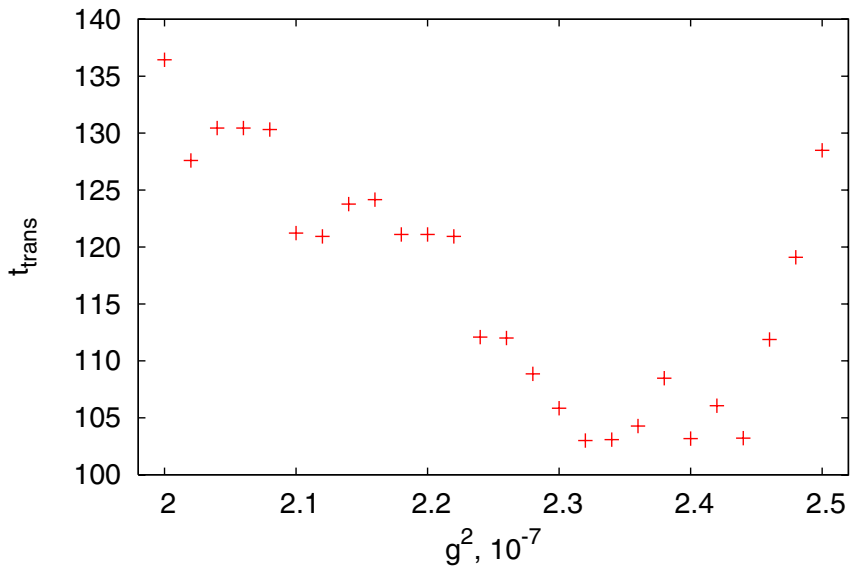

FIG. 2 (color online). Transition (preheating) time as a function of $g^{2}$. 
We see that the transition time varies between $100 / m$ and $150 / \mathrm{m}$. This nonmonotonic behavior of the duration of preheating is explained in the theory of broad paremetric resonance [6] (see Sections 6 and 9 there).

The $g^{2}$ dependence of the EOS is the critical issue for the theory of modulated cosmological perturbations, which we will discuss in Section IV.

(iii) The third point is that $w$ does not necessarily immediately go to the radiation-dominated value $1 / 3$. This is partly because immediately after preheating the light field still has a significant induced effective mass due to the interaction, and partly due to the significant residual contribution from the homogeneous inflaton [14]. Unfortunately, limitations on running longer simulations preclude us from seeing further details of the time evolution of $w$. However, we have a strong theoretical argument to advance the discussion further. In a model with a massive inflaton and light scalar $\chi$ even the radiation-dominated stage is transient. Indeed, sooner or later the massive inflaton particles, even if significantly under-abundant at the end of preheating, will become the dominant component, and the universe will again be matter-dominated.

\section{Occupation numbers}

The occupation numbers $n_{k}^{\chi}$ and $n_{k}^{\phi}$ are among the most interesting variables to understand the microphysics in our system of two interacting fields. First, we shall determine when the definition of $n_{k}$ is meaningful. To do so, we consider the composition of total energy density $\epsilon$ of the system of coupled $\phi$ and $\chi$ fields. The total energy density $\epsilon$ can be decomposed into partial contributions from the kinetic energy of both fields, their gradient energy, their potential energy ( $\phi$ only in this model), and finally the interaction energy

$$
\begin{aligned}
\epsilon= & \frac{1}{2} \dot{\phi}^{2}+\frac{1}{2} \dot{\chi}^{2}+\frac{1}{2 a^{2}}(\nabla \phi)^{2}+\frac{1}{2 a^{2}}(\nabla \chi)^{2}+\frac{1}{2} m^{2} \phi^{2} \\
& +\frac{1}{2} g^{2} \phi^{2} \chi^{2} .
\end{aligned}
$$

Figure 3 shows the relative contribution to the total energy from each of the components for $g^{2}=2.5 \times 10^{-7}$.

We note two features of this plot. First, the interaction term is comparable to the other terms in the time interval $100 / m<t<120 / m$. In this short period, the formula (6) for the energy of the particles is not a good approximation and the occupation number $n_{k}$ is not well defined. Outside this time interval, $n_{k}$ is a meaningful quantity. Secondly, the contribution from the background homogeneous inflaton is dominant even after preheating, up to approximately $t \sim 150 / \mathrm{m}$. A similar point was made for the $\lambda \phi^{4}$ model in [14].

Let us now turn to the occupation numbers $n_{k}$. We find it more instructive to output not the occupation numbers $n_{k}$ per se (as it is commonly done in the literature) but the

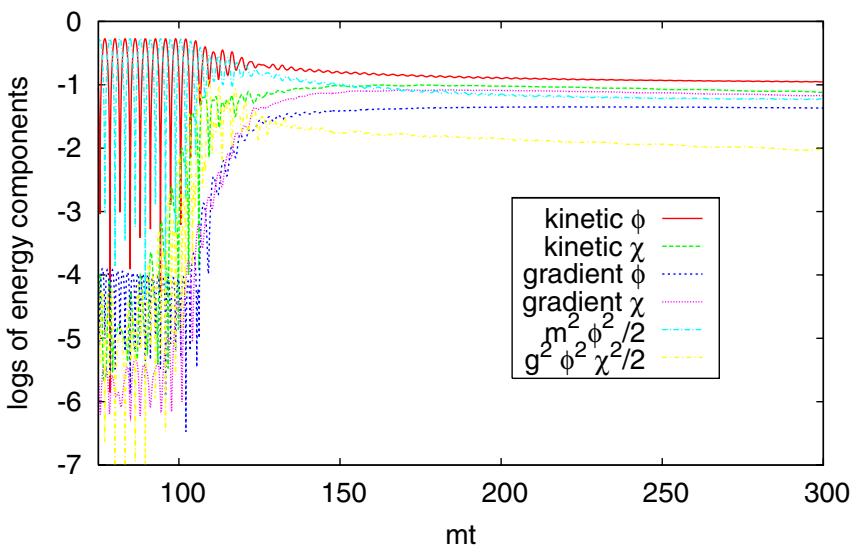

FIG. 3 (color online). Relative contribution of each of the energy components to the total energy, as a function of time. The vertical axis is the log of the various energy components in units of the initial energy $m^{2} \phi_{0}^{2}$ multiplied by $a^{3}$.

combination of $n_{k}$ with the energy per mode $\omega_{k}$. This combination can be immediately compared with the Rayleigh-Jeans spectrum,

$$
n_{k} \approx \frac{T_{\mathrm{eff}}}{\omega_{k}-\mu},
$$

which corresponds to the equipartition spectrum of classical waves (we introduce the chemical potential $\mu$ for generality). The comparison allows to determine how close the distribution is to the thermal one.

The combination $n_{k} \omega_{k}$ for the two fields is shown at some characteristic times in the two Figs. 4 and 5. There are three distinct stages which characterize the evolution covered by our simulation. The first stage, characterized by linear dynamics, is the one of preheating and early rescattering. The first modes to be populated are the IR ones. Preheating of $\chi$ particles occurs in the resonant band at comoving momentum $k_{*}=\sqrt{g m \phi_{0}} a^{1 / 4} \simeq 7 m a^{1 / 4}$ [6].

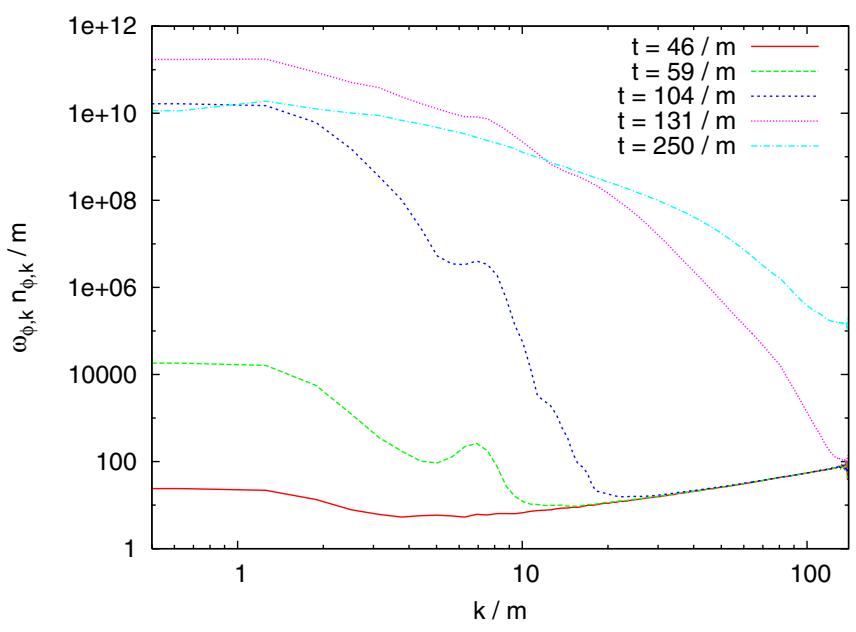

FIG. 4 (color online). Time evolution of the combination $\omega_{\phi, k} n_{k}^{\phi}$, for the model $g^{2}=2.5 \cdot 10^{-7}$. 
EQUATION OF STATE AND BEGINNING OF ...

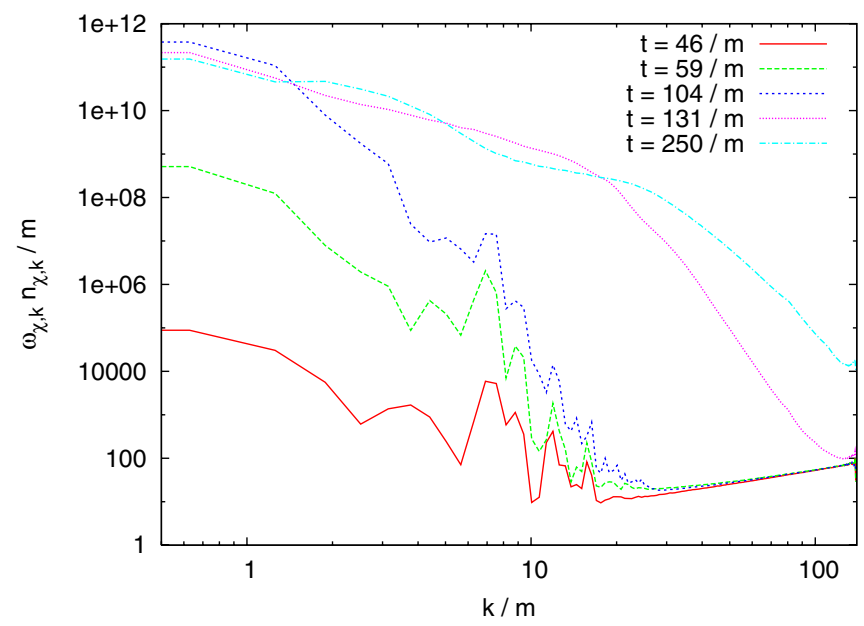

FIG. 5 (color online). Same as Fig. 4, but for the $\chi$ field.

Then, quanta $\delta \phi$ are generated by rescattering. The annihilation $\delta \chi_{k} \delta \chi_{k} \rightarrow \delta \phi_{k} \delta \phi_{k}$ amplifies quanta of the inflaton at $k \simeq k_{*}$. Even more effective is the rescattering of the $\chi$ quanta against the inflaton zero mode, $\delta \chi_{k} \phi_{0} \rightarrow$ $\delta \chi_{k} \delta \phi_{k}$, which produces quanta of both $\phi$ and $\chi$ at momentum $k \simeq k_{*} / 2$.

The second stage is a violent stage of highly nonlinear dynamics. Starting at $m t \sim 100-110$, the higher band at $k_{*} / 2$ both increases in its amplitude and broadens towards higher momenta; quite interestingly, the peak location shifts from $\sim k_{*} / 2$ to $\sim k_{*}$ during this quick and explosive stage. As we remarked, the particle occupation number is ill defined at this stage, and a nonlinear wave description is more adequate.

The explosive stage of rescattering ends at about $m t \sim$ 130. In the next stage, characterized by perturbative dynamics, the distributions smooth out and start evolving (at a much slower rate) towards higher comoving momenta. The spectra in the IR approach a saturated power-law state, which then slowly propagates towards the UV. Although one can observe a greater tendency towards thermalization for the IR modes (where the rescaled spectra are closer to flat), the overall distributions are still typical of the turbulent regime, and they are far from thermal. If we contrast this with the macroscopic behavior we have described above, particularly the evolution of the EOS, we see that the system can be considered in a prethermalized state (see also [18]), but that thermalization is still far from being complete .

It is also instructive to consider the product of the occupation number $n_{k}$ with the phase space sphere area $k^{2}$ and energy per mode $\omega_{k}$. This combination represents the energy density of the quanta at momentum $k$ (since the product $4 \pi k^{2} \omega_{k} n_{k} d k$ is the energy density of the quanta whose momentum has a magnitude between $k$ and $k+d k$ ).

In Figs. 6 and 7, we plot the distributions at different times separated by intervals $\delta t=4 \pi / m$. This allows us to visualize the growth of the distributions, and to monitor the
PHYSICAL REVIEW D 73, 023501 (2006)

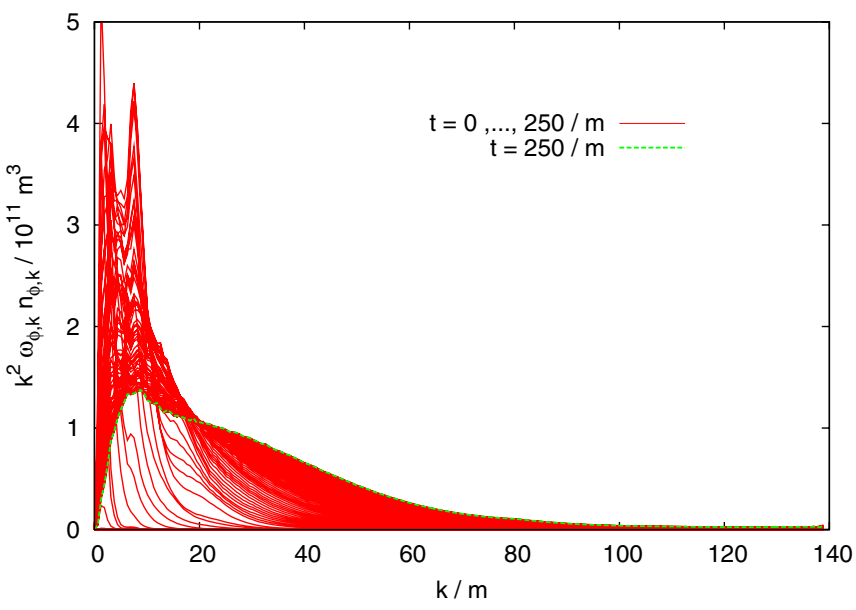

FIG. 6 (color online). Time evolution of the combination $k^{2} \omega_{k} n_{k}$ for the field $\phi$. The thicker curve is the spectrum at the final time of our evolution.

cascade of energy in the phase space $k$. When the ultraviolet part of the distribution hits the highest momentum of the simulation (defined by the grid size), the energy is artificially reflected back to the IR modes and the simulation is no longer reliable. Due to the scale chosen (natural rather than log scale), only the distributions at times greater than about 100/ $m$ can be appreciated in the two Figs. 6 and 7 . Moreover, the double peak structure that can be observed for $\chi$ at late times is due to the rescaling chosen. The plot for the occupation number, $n_{k} k^{2}$ (not shown) has a high peak at $k \sim 4 m$, followed by a plateau up to $k \sim 30 m$. The combination $k^{2} \omega_{k} n_{k}$ shown has a peak at the momenta corresponding to this plateau, showing that these momenta dominate the energy density in the $\chi$ distribution. The saturated spectra can be clearly seen in the thick curves on these plots.

The combination $k^{2} \omega_{k} n_{k}$ is also given in Figs. 8 and 9, where we however show only few times, and we focus on the IR part of the distributions. These figures show the

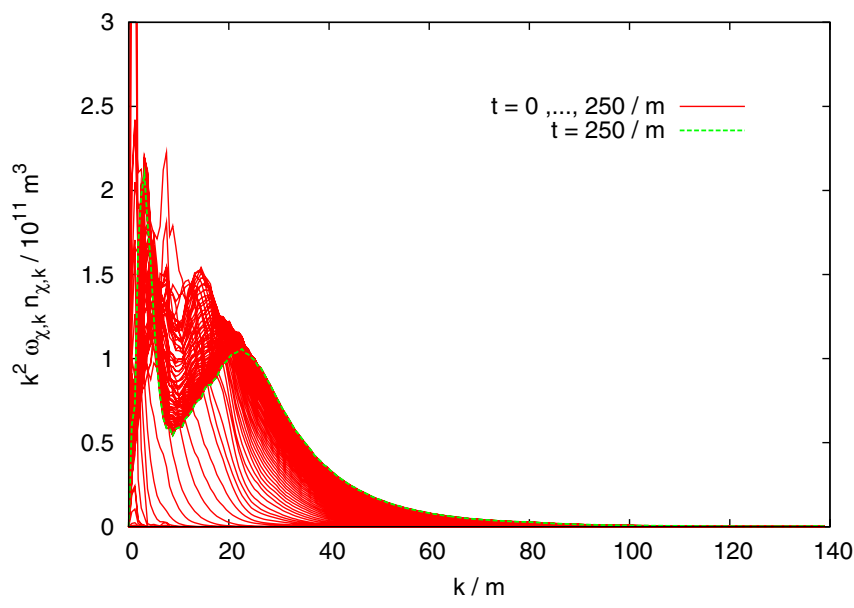

FIG. 7 (color online). Same as Fig. 6, but for the field $\chi$. 


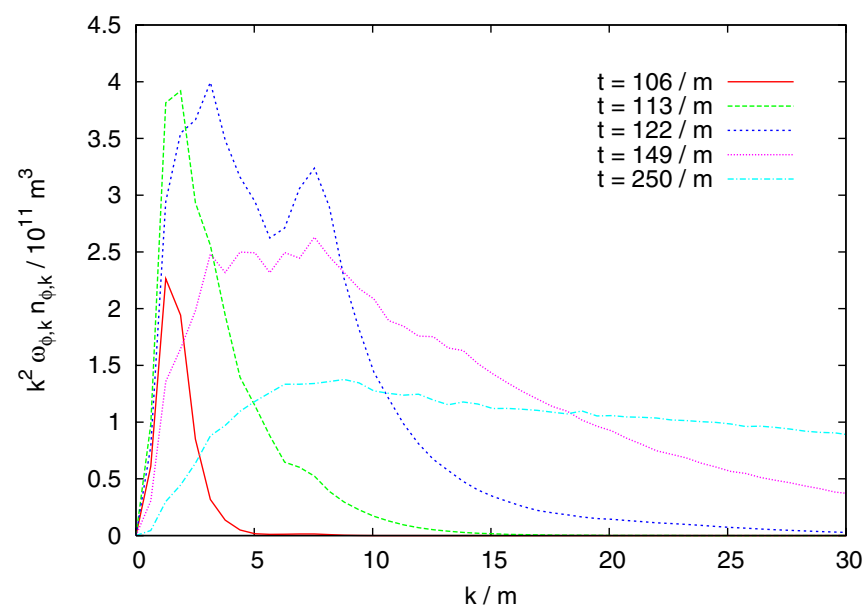

FIG. 8 (color online). Same combination as in Fig. 6, but zoomed in the IR region.

rapid broadening (towards the UV) of the distributions in the violent rescattering stage, and the tendency to saturation at later times.

\section{Fluctuations and effective masses}

The evolution of the scalar fields can be strongly affected by the presence of dynamical effective masses for the two fields. A high mass for some of the fields can disfavour or block some of the interactions, with a consequent delay of thermalization. For instance, it is wellknown that a condensate which is strongly coupled to some field $\psi$ can have a very long lifetime, since the same coupling which would allow the decay also produces a high effective mass for $\psi$, which can prevent the decay from occuring. In the case we are discussing, the effective masses of the fields acquire significant (loop) contributions from the high variances produced at preheating/rescattering, according to Eqs. (8) and (9).

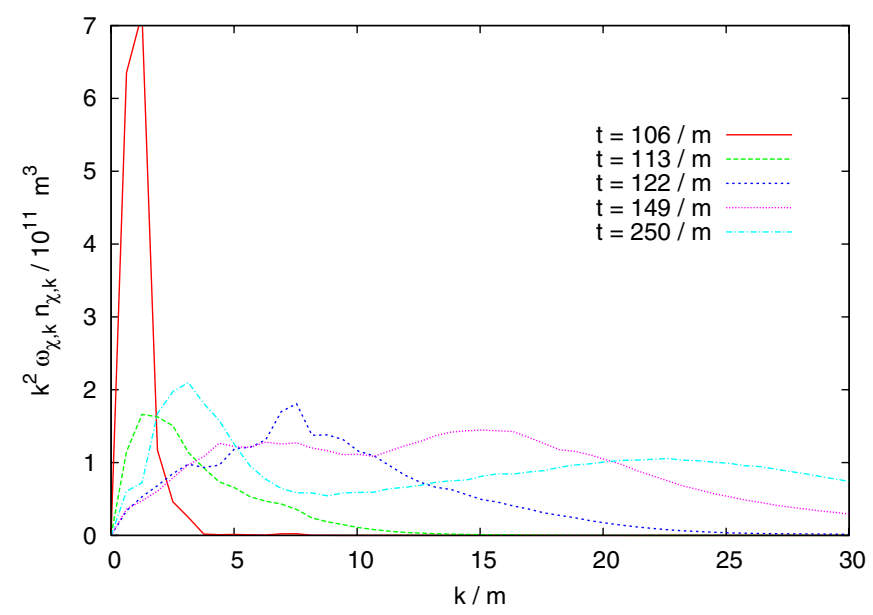

FIG. 9 (color online). Same as Fig. 8, but for the field $\chi$.

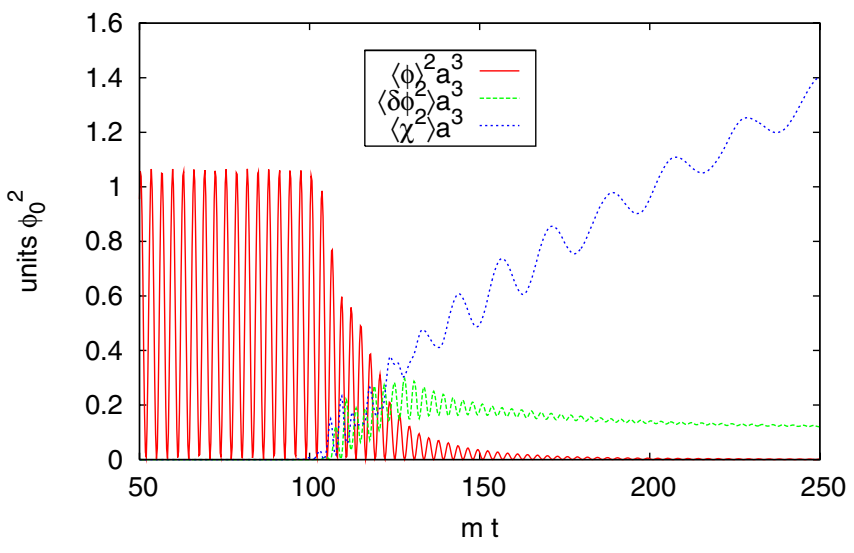

FIG. 10 (color online). Evolution of the background inflaton field $\langle\phi\rangle^{2}$ and of the variances $\left\langle\delta \phi^{2}\right\rangle$ and $\left\langle\chi^{2}\right\rangle$. Fields squared are rescaled by $a^{3}$.

Figure 10 shows the evolution of the background inflaton oscillations and fluctuations of $\left\langle\delta \phi^{2}\right\rangle$ and $\left\langle\delta \chi^{2}\right\rangle$. All values are plotted in "comoving scales", i.e. multiplied by $a^{3}$. The corresponding physical quantities can be obtained by the value of the scale factor, which is shown in Fig. 11.

Figure 12 shows the effective masses for $\phi$ and $\chi$, multiplied by the scale factor $a$. This combination enters in the comoving dispersion relations for the quanta of the two fields, given by Eq. (7). Therefore, the product $a m_{\mathrm{eff}}$ is the correct quantity to compare with the comoving momentum $k$.

This comparison is shown in Fig. 13, where we show the fraction of relativistic quanta (that is, with $k>a m_{\mathrm{eff}}$ ) for the two fields. We see that the majority of the particles are nonrelativistic, so that the effective masses indeed play a significant role in the evolution of the spectra. In particular, we observe that during rescattering very few particles are relativistic; this confirms the fact that the distributions are very peaked in the IR at this stage. The fraction of relativistic $\chi$ particles rapidly increases during the violent ther-

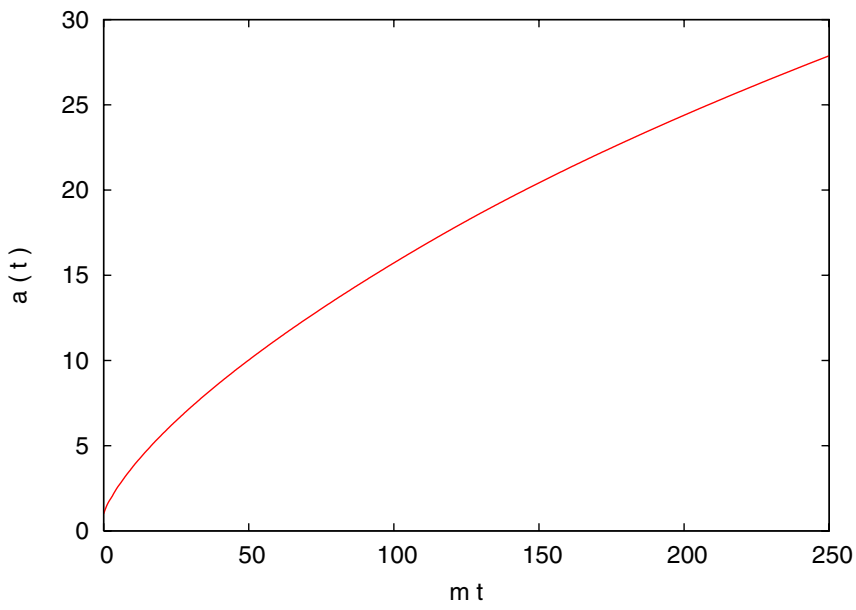

FIG. 11 (color online). Evolution of the scale factor $a(t)$. 


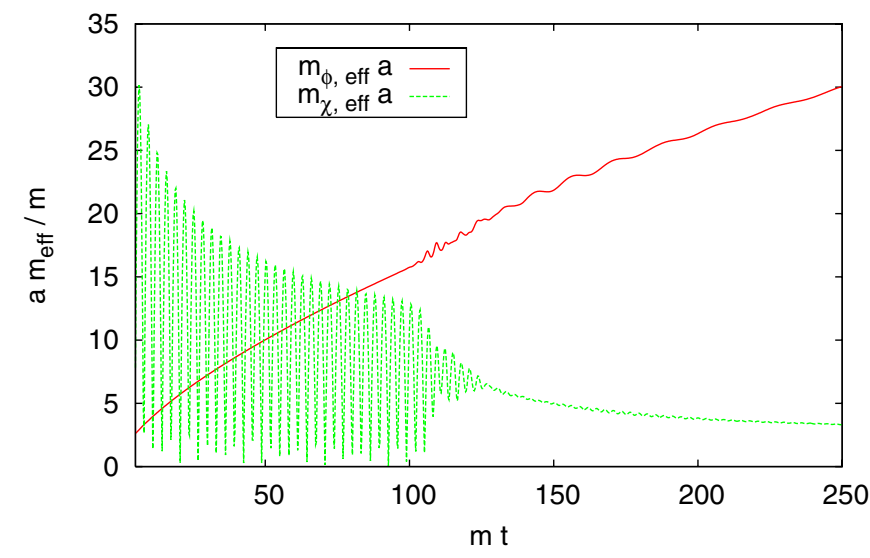

FIG. 12 (color online). Evolution of effective masses multiplied by scale factor $a$ (as it is relevant for the comoving frequency (7)).

malization stage. The fraction of relativistic $\delta \phi$ remains instead always smaller (confirming the fact that $m_{\phi, \text { eff }}>$ $m_{\chi, \text { eff }}$ ), and it slowly increases during the thermalization stage, when the distributions evolve towards the UV.

Up to $m t \sim 120$, the coherent mode of the inflaton dominates the effective mass for $\chi$. (The rapid variation of $m_{\chi \text {,eff }}$ due to the coherent oscillations of $\phi$ is the cause of preheating.) At later times, the variance of $\phi$ gives instead a greater contribution. In the effective mass for $\phi$ the "bare" term $m$ dominates up to $m t \sim 100$, while the variance of $\chi$ provides the dominant contribution at later times. Equation (12) provides a useful expression for the variance; during the turbulence stage, the occupation numbers vary only adiabatically, so one may expect that the growth of $a m_{\text {eff }}$ results in a decrease of the variance, and vice-versa. This relation is visible in the figures shown. The "late time" decrease of $a m_{\chi, \text { eff }}$ is accompanied by an increase of $\left\langle\chi^{2}\right\rangle$. This in turns cause an increase of $a m_{\phi, \text { eff }}$, and, consequently, a decrease of $\left\langle\phi^{2}\right\rangle$.

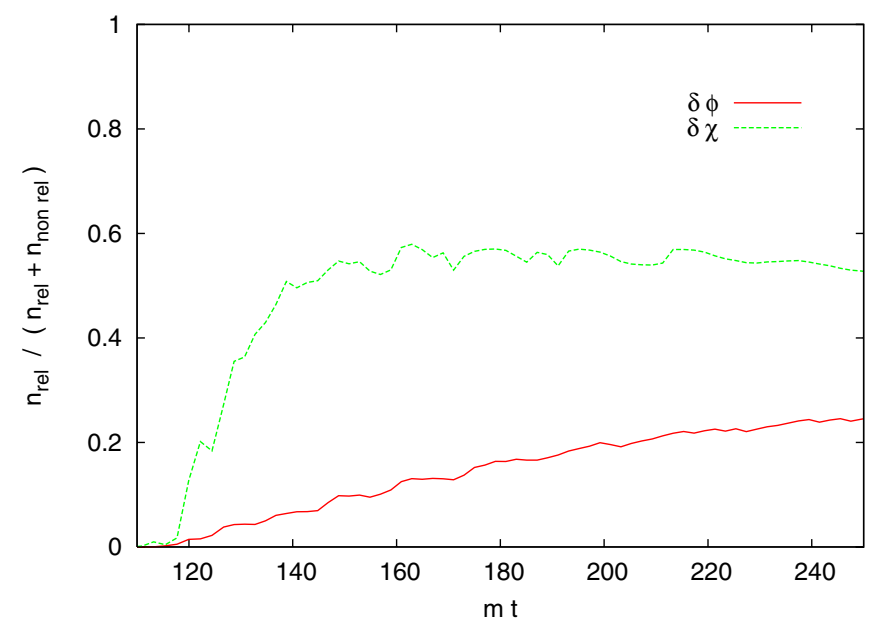

FIG. 13 (color online). Fraction of particles in the relativistic regime, $k>a m_{\mathrm{eff}}$.

\section{COSMOLOGICAL IMPLEMENTATIONS OF THE RESULTS}

\section{A. Complete decay of the inflaton}

In the simple model discussed so far, with the interaction term $\phi^{2} \chi^{2}$, the decay of the inflaton $\phi$ is not complete. Indeed, such a term mediates scatterings between inflaton quanta, rather than single particle decay. Once $\phi$ is diluted by the expansion of the universe, the scatterings become inefficient, and the number of inflaton quanta remain practically constant. This poses a problem because in order to reach the stage of radiation domination we need to have complete decay of the inflaton.

The simplest way to have a complete decay is to consider three-legs interactions. For instance, we can replace (3) by

$$
\mathcal{L}_{\mathrm{int}}=-\frac{g^{2}}{2}(\phi+\sigma)^{2} \chi^{2},
$$

where $\sigma$ is a mass dimension parameter, which breaks the $\phi \leftrightarrow-\phi$ symmetry. For $\sigma \ll \phi$, the trilinear term produced by this interaction is irrelevant and the inflaton decay occurs as described above. However, when $\phi$ is decreased by the expansion of the universe, the trilinear interaction becomes dominant, leading to a complete inflaton decay.

We can avoid discussing the introduction of the scale $\sigma$ by considering a Yukawa interaction of the inflaton with some fermions $\psi$. By itself, this interaction has interesting consequences for preheating, as discussed e.g. in [24]. When both fermionic and bosonic interactions are present, preheating into bosons is typically much more efficient, since the one into fermions is reduced by Pauli blocking. However, once the interactions become perturbative, the trilinear $\phi \rightarrow \psi \psi$ decay will eventually dominate over the $\phi \phi \rightarrow \chi \chi$ scattering.

Three-legs interactions arise very naturally in SUSY theories. Indeed, consider the simple superpotential

$$
W=\frac{m}{\sqrt{8}} \phi^{2}+g^{2} \phi \chi^{2} .
$$

The corresponding scalar field potential contains three-legs as well as four-legs plus self-interaction terms

$$
V=\frac{m^{2}}{2} \phi^{2}+\sqrt{2} g^{2} m \phi \chi^{2}+4 g^{2} \phi^{2} \chi^{2}+g^{4} \chi^{4}
$$

(neglecting the imaginary parts of the fields). The four-legs interaction dominates over the three-legs one as long as $\phi \gtrsim \sigma=2 g^{2} m$. For reasonable values of $\sigma$, this happens during the first stages of preheating. Eventually, the amplitude of $\phi$ decreases due to the expansion of the universe, and the trinlinear interaction dominates, resulting in a complete decay of the massive inflaton.

Hence, considering a supersymmetric theory automatically introduces trilinear vertices among the scalars. It also allows for stronger couplings between the inflaton and 
other fields, without spoiling the flatness of the inflaton potential, as we have discussed in Sec. II.

\section{B. Relation between e-foldings $N$ and wavelength of perturbations}

The precise history of the expansion of the universe $a(t)$ is important for connecting the physical wavelength $k / a(t)$ of the cosmological perturbations we observe to the number of e-foldings $N$ at which the perturbation was generated (left the horizon) during inflation.

According to the general lore $[25,26]$, one has

$$
N(k)=62-\ln \frac{k}{6.96 \times 10^{-5} \mathrm{Mpc}^{-1}}+\Delta,
$$

where $6.96 \times 10^{-5} \mathrm{Mpc}^{-1}$ is the inverse size of the present cosmological horizon and $\Delta$ is defined by the physics after inflation:

$$
\Delta=-\ln \frac{10^{16} \mathrm{GeV}}{V_{k}^{1 / 4}}+\frac{1}{4} \ln \frac{V_{k}}{V_{\text {end }}}-\frac{1}{3} \ln \frac{V_{\text {end }}^{1 / 4}}{\rho_{\text {reh }}^{1 / 4}},
$$

where $\rho_{\text {reh }}$ is the energy density at the end of reheating, $V_{k}$ is the value of the inflaton potential at the moment when the mode with the comoving wave number $k$ exits the horizon at inflation and finally $V_{\text {end }}$ is the value at the end of inflation.

The last term in the right hand side of Eq. (21) is related to reheating, specifically that it is not an instantaneous process. It is typically computed under the assumption that the universe is matter-dominated (by the coherent oscillations of the inflaton) all throughout reheating, and that it (instantaneously) becomes radiation-dominated at the end of the reheating stage. As we have seen, this is not what happens.

Indeed, a better definition of the last term in $\Delta$ is $-\frac{1}{4} \times$ $\ln \left(a_{\mathrm{rd}} / a_{\text {inf }}\right)$ where $a_{\mathrm{rd}}$ is the scale factor at the moment after reheating at which the EOS reaches its value at radiation dominance. As we have been discussing, this moment can occur while the distributions of particles are very far from thermal, shortly after the end of preheating. This amounts to taking $a_{\mathrm{rd}} / a_{\text {inf }} \sim 10-20$, rather than $a_{\mathrm{rd}} / a_{\mathrm{inf}} \gtrsim 10^{8}$, where $a_{\mathrm{RD}}$ is the value of the scale factor at the end of reheating under the common assumption that perturbative reheating occurs entirely within a regime of matter domination, with $T_{r} \simeq 10^{9} \mathrm{GeV}$. In this situation one has $H_{\mathrm{RD}} \sim T_{r}^{2} / M_{P}$ at the end of matter domination, and the corresponding value of the scale factor $a_{\mathrm{RD}}$ can be estimated as $a_{\mathrm{RD}} / a_{\text {inf }} \sim\left(H_{\text {inf }} / H_{\mathrm{RD}}\right)^{2 / 3} \sim\left(m M_{P} / T_{r}^{2}\right)^{2 / 3}$.

Hence, for a preheating scenario with a very wide range of values of the coupling $g^{2}$, the first term of (21) can be fixed to

$$
-\frac{1}{4} \ln \frac{a_{\mathrm{rd}}}{a_{\mathrm{inf}}} \sim-(0.6-0.8) .
$$

This result holds for a wide class of inflationary models, and for many different types of preheating.

\section{Physics before thermalization}

One of the most important parameters in physics of the early universe is the highest temperature of the hot plasma after the inflaton field decays and transfer its energy into radiation. $^{2}$ This is traditionally called the reheating temperature $T_{r}$. As we have seen in many previous papers and above in this paper, ultimate thermal equilibrium is preceeded by several stages. Let us distinguish four of them:

(i) preheating, with the duration $\delta t_{1} \sim 100 / \mathrm{m}$,

(ii) a short violent stage at the end of preheating when nonlinearity and nonperturbative effects are dominant; chaos is onset, erasing the details of the initial conditions, on a timescale $\delta t_{2} \sim 10 / \mathrm{m}$,

(iii) the stage of turbulence of classical fields, with the saturated spectrum cascading both towards UV and IR modes with duration $\delta t_{3}$, longer than the two previous stages,

(iv) the last stage of proper QFT thermalization, with particle fusion/offshell processes; it is characterized by the timescale $\delta t_{4}$, much longer than the previous ones.

Traditionally the beginning of the thermal history is related to the moment $\delta t_{4}$, which may be very long; as a result of redshift, the reheating temperature can be rather low.

Let us reconsider this attitude. While the question of when the ultimate thermal equilibrium will be established and to which value of $T_{r}$ it corresponds is still very interesting, we will argue that important physics which constrains the model of inflation and interactions takes place long before full thermal equilibrium is completed.

Indeed, Figs. 4-6 show that the modes at physically interesting scales, up to $k \sim$ tens of $m \sim 10^{14} \mathrm{GeV}$, are quickly excited towards a saturated distribution. This raises several subtle and interesting questions. Usually, the relativistic particles embedded in the thermal bath are brought into thermal equilibrium after the relaxation time $1 / \sigma n$, where $\sigma$ is the cross section of the processes relevant for thermalization, and $n=\int d^{3} k n_{k}$ is the number density of particles. This estimate works in the diluted gas approximation. Indeed, consider the vertex $\phi \chi^{2}$, leading to inflaton decay into quanta of $\chi$. The particle number density enters in the collisional integral in the combination

$$
n_{p 1}^{\phi}\left(1+n_{p 2}^{\chi}\right)\left(1+n_{p 3}^{\chi}\right)-n_{p 2}^{\chi} n_{p 3}^{\chi}\left(1+n_{p 1}^{\phi}\right)
$$

\footnotetext{
${ }^{2}$ It is well-known that if you assume a slow, perturbative inflaton decay and a quick thermalization of the decay products, a subdominant thermal bath will be present while the inflaton is still decaying. That will have a temperature muich higher than $T_{r}$, defined at the moment when the thermal bath starts dominating.
} 
( $p_{1}$ is the momentum of the inflaton quantum, while $p_{2,3}$ are the momenta of the two quanta of $\chi$ entering in the process). The above estimate for the thermalization timescale assumes that the inverse process $\chi \chi \rightarrow \phi$ (described by the second term in (23)) is irrelevant, and that all the occupation numbers are much smaller than 1 , so that $1+$ $n_{p} \simeq 1$. In particular, this last assumption is not valid until the very end of the thermalization stage.

Therefore, for large $n_{k}$ we expect that particles are dragged into effective thermal equilibrium faster, due to the stimulated interaction. We have observed this fact also in previous numerical lattice simulations [13].

Another relevant effect, already discussed above, is that large fluctuations of fields can contribute to the effective masses of particles, which can become effectively heavy. This leads to the blocking of some processes which may lead to a faster thermalization, until fields fluctuations are diluted by the expansion.

Finally, a very relevant question is the one of particle production from the nonthermal but highly excited distributions. In particular, one should verify that dangerous relics are not overproduced at this stage. To see this, let us discuss gravitino production from the decay products of the inflaton. In models where supersymmetry is broken gravitationally, only a very small number of gravitinos can be tolerated, $Y_{3 / 2}=n_{3 / 2} / s \lessgtr 10^{-14}$ (the exact value being dependent on the gravitino mass) [20]. ${ }^{3}$ The typical approach is to compute the production of gravitinos only after the thermal bath has formed. The dominant processes are $2 \rightarrow 2$ scatterings with only one gravitino as outgoing particle (and, hence, only one gravitationally suppressed vertex, while the other vertex is typically a gauge interaction). The rate of gravitino production follows from the Boltzmann equation

$$
\frac{d N_{3 / 2}}{d t}+3 H N_{3 / 2}=\langle\sigma|v|\rangle N_{T} N_{T}
$$

where $N_{T} \sim T^{3}$ is the abundance of the (MSSM) degrees of freedom in the thermal bath, and where the average cross section is of the order $\langle\sigma|v|\rangle \sim \alpha / M_{p}^{2}$, where $\alpha$ is a gauge structure constant. The right hand side decreases very rapidly with time, and the production at the highest possible temperature (that is, at $T_{r}$ ) dominates. Hence, only the production in the first Hubble time is relevant, and the final result can be estimated as

$$
N_{3 / 2} \sim \frac{\alpha T_{r}^{6}}{M_{p}^{2}} \times H_{r}^{-1}
$$

where $H_{r} \sim 10 T_{r}^{2} / M_{p}$ is the Hubble parameter at $T=T_{r}$. This leads to the gravitino abundance

\footnotetext{
${ }^{3} \mathrm{~A}$ more stringent limit applies if the decay has a significant hadronic branching ratio [27].
}

$$
Y_{3 / 2} \sim 10^{-4} \frac{T_{r}}{M_{p}}
$$

and to the well-known bound $T_{r} \lesssim 10^{9} \mathrm{GeV}$ on the reheating temperature.

This standard computation of gravitinos generated from the thermal bath neglects all the production which may have taken place during the thermalization stage. The underlying idea is that, during reheating, most of the energy is in the inflaton condensate, where the quanta (loosely speaking) do not have momentum, and hence cannot scatter to produce gravitinos. While this may be true for a slow (perturbative) inflaton decay, this is certainly not the case for preheating/rescattering, where the distributions of the decay products form right after the end of inflation. These distibutions may be responsible for significant gravitino production, even before complete thermalization has taken place [28].

It is worth emphasizing here that a fully quantum treatment of the gravitinos production is not in order. For instance, Refs. [17,18] discuss thermalization of fermions (in a Minkowski universe) through a loop expansion of the 2PI effective action. Loop effects for the gravitino case are gravitationally suppressed, and can be safely neglected in the present computation. Once a gravitino is produced, it simply propagates freely (without any modification in its distribution function), and with only gravitational interaction with the rest of the theory, until it decays (if unstable). There is a crucial distinction between the longitudinal and the transverse gravitino components, see for instance [19]. At any given time, the longitudinal gravitino component is provided by the superpartner(s) of the field(s) responsible for supersymmetry breakdown. In the early stages of thermalization, supersymmetry is mostly broken by the inflaton decay products, so that the longitudinal gravitino component at that time is coupled to these fields more strongly than gravitationally. However this component is not relevant for the gravitino problem, and we are not interested in computing its abundance (this field is abundantly produced, but it decays well before nucleosynthesis, when it is not anymore associated with the longitudinal gravitino). In models where supersymmetry is broken in a hidden sector, and where the breaking is communicated to the observable sector through gravity, the relevant (late time) longitudinal gravitino component is always interacting only gravitationally with fields in the observable sector, and the estimate below applies. In addition, the transverse gravitino component does not share this problem, and its interaction with the observable sector is gravitational at all times. To avoid excessive model dependency, the consideration below focus on the transverse component. However, as we mention, in many models they also apply to the longitudinal component.

The computation of the gravitino abundance is more difficult than in the thermal case [20]. First of all, unlike the thermal computation, the evolution of the bosonic 
fields (responsible for the production) is model dependent. Second, turbulent nonlinear processes are dominant right after preheating, so that it is possible that processes including more vertices give a sizeable contribution to the amount of gravitinos produced. However, we can obtain a conservative estimate by considering only $2 \rightarrow 2$ tree level processes also in this case. For definiteness, let us take the model (1) and (3), with $g^{2}=2.5 \times 10^{-7}$ (the value that we have studied above), and assume that the $\chi$ field produced at rescattering has a trilinear vertex with its femionic superpartner $\tilde{\chi}$ and another fermion $\tilde{z}$. For instance, this could be a gaugino, if $\chi$ has gauge interactions. However, let us consider here a generic vertex with coupling constant $h$ and the interaction $\chi \chi \rightarrow \psi_{3 / 2} \tilde{z}$, mediated by the fermionic partner of $\chi$.

We are interested in evaluating the production at the time $t_{*} \sim 120 / m$, when the (physical) number density of quanta of $\chi$ is maximal. We first discuss whether the process we are considering is kinematically allowed. At the time $t_{*}$, the nongravitational vertex provides an effective mass for fermions of the order (see Fig. 10).

$$
m_{\tilde{z}}\left(t_{*}\right) \sim h \sqrt{\left\langle\chi^{2}\left(t_{*}\right)\right\rangle} \sim 5 \cdot 10^{2} h m
$$

The distributions of $\chi$ obtained from the lattice have a typical comoving momenum of the order of $10 \mathrm{~m}$, which, at the time $t_{*}$, corresponds to the physical momentum $p_{*} \sim$ $0.5 \mathrm{~m}$. Hence, if $h<10^{-3}$, most of the quanta of $\chi$ will be able to produce gravitinos through the process we are considering. For higher values (for example, if the second vertex is a gauge interaction), only the few quanta of $\chi$ with very high momenta can contribute to the production, so that this process is significantly suppressed. However, as the universe expands, $\sqrt{\left\langle\chi^{2}\right\rangle}$ decreases as $a^{-3 / 2}$, while the physical momenta $p \propto a^{-1}$ decrease less. Therefore, more and more quanta will have a sufficiently high momentum to produce a $\psi_{3 / 2}-\tilde{z}$ pair. ${ }^{4}$ For simplicity, $h \leqq 10^{-3}$ is assumed in the following discussion; we do not expect that the following conclusions will significantly change if a stronger coupling is considered.

By proceeding as in the thermal case, we can estimate the number of gravitinos produced in the first Hubble time after $t_{*}$. (Again, the numerical values are taken from the lattice results; $N_{\chi}\left(t_{*}\right)^{2}$ denotes the physical number density of the $\chi$ quanta at the time $t_{*}$.)

$$
N_{3 / 2}\left(t_{*}\right) \sim \frac{h^{2}}{M_{p}^{2}} N_{\chi}\left(t_{*}\right)^{2} H\left(t_{*}\right)^{-1} \sim 10^{7} h^{2} m^{3} .
$$

For sufficiently high $h$, this value is actually incorrect,

\footnotetext{
${ }^{4}$ This scaling argument does not include the effect of the thermalization, which is also increasing the $p / m_{\tilde{z}}$ ratio. Indeed, since thermalization proceeds through particle fusion it has the effect of both increasing the typical momenta of the distributions and of decreasing the effective masses.
}

since it exceeds Pauli blocking. Assuming that the whole Pauli sphere up to physical momentum $p_{*}$ is filled gives

$$
N_{3 / 2}\left(t_{*}\right) \sim 0.1 m^{3} .
$$

The number density of produced gravitinos is then the smaller between Eqs. (28) and (29), according to the value of $h$. The expression (29) is relevant only for $10^{-4} \lesssim h \lesssim$ $10^{-3}$; therefore, since we are interested in an upper limit for $h$, we will consider the value given by (28) in the following.

The gravitino abundance is obtained by dividing the gravitino number density by the entropy. At the time $t_{*}$, the distributions of the decay products are far from thermal (being concentrated in the IR), so that their entropy is smaller than that of a thermal bath with the same energy. The entropy density can be computed from the lattice simulations to be approximately $s=10^{4} \mathrm{~m}^{3}$ at this stage [29]. However, the (comoving) entropy is expected to increase due to thermalization. If we convert the actual particle distribution instanteneously into the thermal distribution, the entropy would reach the maximal value $s_{\max } \sim 10^{7} \mathrm{~m}^{3}$ allowed by energy conservation. The difference between the actual entropy density and $s_{\max }$ gives rise to the dilution of the gravitino abundance during thermalization. (Further sources of dilution require extensions of the minimal model.)

Therefore, the conservative estimate for the gravitino abundance is obtained by dividing the gravitino number density by

$$
\rho\left(t_{*}\right)^{3 / 4} \sim 10^{7} \mathrm{~m}^{3} .
$$

where $\rho$ is the (physical) background energy density at the time $t_{*}$. Hence, the ratio

$$
Y_{3 / 2}\left(t_{*}\right)=\frac{N_{3 / 2}\left(t_{*}\right)}{\rho^{3 / 4}\left(t_{*}\right)} \sim h^{2}
$$

Therefore, the nucleosynthesis bound results in the strong upper bound $h \lesssim 10^{-7}$.

This is a serious concern, which has to be addressed in the model building of inflation. It can be resolved by introduction of extra radical assumptions. For instance, this gravitino abundance can be decreased by an entropy dilution. This happens if a massive species $\psi$ is produced at reheating, and it dominates the energy density of the universe for some time. When it decays, it leads to a significant amount of entropy, which dilutes the particle pieces produced directly at the inflaton decay. Concretely, the entropy dilution leads to the decrease of the gravitino abundance $Y_{3 / 2} \sim N_{3 / 2} / \rho^{3 / 4}$. Supposing that the universe is matter-dominated between the times $t_{1}$ and $t_{2}$, the ratio $N_{3 / 2} / \rho^{3 / 4}$ is decreasing as $\left(a_{1} / a_{2}\right)^{3 / 4}$ ( $a_{i}$ being the scale factor at the time $t_{i}$ ). This entropy dilution could also occur through a secondary stage of inflation [30]. 
It should be clear that the result (31) is very sensitive to the specific model considered, and that a case by case calculation is necessary.

\section{Modulated fluctuations from preheating}

One of the motivations for studying the EOS after preheating is related to the theory of modulated cosmological fluctuations. We briefly review the idea as it was presented in $[8,9]$, and discuss the implications of our findings on the evolution of the EOS for this mechanism. We leave the explicit calculations for subsequent publication.

Suppose that the coupling $g^{2}$ depends on some modulus field $z$, (as is typical in string theory)

$$
g^{2}=g^{2}(z), \quad \delta g^{2}=\frac{d g^{2}}{d z} \delta z
$$

During inflation the modulus field $z$ can be light, with a mass smaller than the Hubble parameter during inflation $H \sim 10^{13} \mathrm{GeV}$. Then, large scale fluctuations $\delta z$ with an almost scale free spectrum are inevitably generated from inflation. The wavelength of fluctuations exceeds the size of the causal patch $H^{-1}$. As we have discussed above, the EOS varies very quickly already at the beginning of the thermalization stage. The variation is a nonmonotonic function of the coupling $g$, see for instance Figs. 1 and 2. Due to the large scale spatial fluctuations of the coupling $\delta g^{2}$, the change in the EOS proceeds with a slight time shift in different Hubble patches. This results in the generation of (almost) scale free scalar metric cosmological perturbations.

To perform accurate calculations of the amplitude of modulated fluctuations, one needs to know the exact character of the transition from inflaton field to radiation. In the simplified picture of perturbative reheating, when the inflaton energy decays exponentially as $\epsilon_{\phi} \sim e^{-\Gamma t}$, calculations of modulated fluctuations [9] are based on linearization of the inflaton decay rate $\Gamma$, which is linearly dependent on the coupling $g^{2}$. However, as we have remarked, the decay of the inflaton field is typically more complicated, and the transition towards RD occurs very quickly (well before thermalization completes). In addition, we stress that the time of the transition is a nonmonotonic function of $g^{2}$. Finally, although we focused only on the direct coupling of the inflaton to matter, the details of thermalization (and of the evolution of the EOS) depend on the other interactions among the decay products; the strength of these interactions are likely to be dependent on some modulus field as well, and they can also modulate cosmological perturbations. All these features have to be taken into account for precision computations of modulated fluctuations.

\section{SUMMARY}

In this paper we studied the out-of-equilibrium nonlinear dynamics of the fields during and after preheating. This continues earlier works in $[13,14,18]$. We use lattice numerical simulations of fully nonlinear dynamics of interacting classical fields. In this investigation, we computed the evolution of the equation of state during preheating and the early thermalization stage. Immediately after inflation, the EOS is the one corresponding to inflaton domination. For a massive inflaton, we have matter domination, characterized by $p=0$. After a very short time, the inflaton transfers its energy into inhomogeneous modes. At the first stage (preheating) inhomogeneous field fluctuations are copiously produced in the regime of parametric resonance. Then the modes rescatter to redistribute the energy by cascading in the phase space. This process of classical wave turbulence can be rather long. This leads to a final stage, where the occupation numbers are small and the classical approximation breaks down. Thermalization finishes when the quantum fields reach the ultimate thermal equilibrium. If all the participating fields are light, the final thermalized state corresponds to an EOS of radiation, with $p=\frac{1}{3} \epsilon$.

The transition between the matter and radiationdominated EOS is hence typically supposed to occur on a (relatively) very long timescale. What we found in our numerical simulations is very different from this naive expectation. In fact, the EOS jumps from matter domination to (almost) radiation domination immediately after the first stage of preheating, i.e. in a couple dozen inflaton oscillations $\left(10^{-37} \mathrm{sec}\right.$ each), long before thermal equilibrium has been established. In other words, the macroscopic EOS is close to the one of radiation domination, while the microscopic state is far from thermal. This result is similar to the conclusion of [18] regarding $O(N)$ theory in flat spacetime.

The sharp change of the EOS we observed can be very conveniently related to the moment of energy transfer from the inflaton to the inhomogeneous radiation. As we mentioned, it is a very fast process, as is well-known from the theory of preheating, which takes place in only about $10^{-35}$ sec. Moreover, the time at which the transition occurs depends only weakly on the coupling $g^{2}$ between the inflaton and the other fields. The exact dependence is nonmonotonic, and the transition time oscillates around $\sim 10^{-35} \mathrm{sec}$ (as set by preheating) for a very wide range of couplings. This result is drastically different from perturbative inflaton decay, where the transition timing is proportional to the coupling.

This very quick evolution has several consequences, and leads to a number of issues which deserve further investigation. We stress four of them.

(i) The first is related to the generation of modulated cosmological fluctuations from preheating. It is conceivable [8-10] that the strength of the inflaton 
interactions with its decay products is a function of some moduli fields as expected in string theory. Fluctuations of the light moduli (with mass smaller than $10^{13} \mathrm{GeV}$ ) are inevitably generated during inflation. This results in spatial variations of the couplings at large scales, well beyond the size of the causal (Hubble) patch at the end of inflation. Hence, the transition from inflation to radiation domination occurs at slightly diffferent times in different Hubble patches, giving rise to scalar metric fluctuations.

To calculate metric fluctuations from modulated perturbations, one needs to know the exact evolution of the EOS, from the matter domination (inflaton) to the radiation domination (light decay products) stage. We found that the transition of the EOS occurs sharply in a steplike manner. Therefore one can introduce a timelike (but spatially rippled) hypersurface which divides two cosmological regimes, with $p=0$ and $p=\frac{1}{3} \epsilon$. Metric fluctuations can be calculated using GR matching conditions across this hypersurface, similar to how it was done to calculate modulated fluctuations in hybrid inflation [10].

(ii) The second application of the EOS history is related to the formula which links the number of efoldings during inflation $N$ and the present day physical wavelengths of fluctuations in terms of the $\log s$ of their momenta $\log k$. Indeed, the cosmological evolution of the scale factor $a(t)$ is ultimately defined by the EOS. Refining the $N-$ $\log k$ relation is mandatory to put precise observational constraints on inflationary models. In this paper we notice that a (practically) immediate transition from inflation to radiation domination is a common feature of inflationary models with preheating, either parametric resonance or tachyonic ones. Equation (21), with our result (22), refines the $N-\log k$ relation.

(iii) The next note is related to the model building of inflaton interactions. In the majority of papers discussing inflaton decay, interactions with bosons are considered in the form of four-legs vertices of the type $g^{2} \phi^{2} \chi^{2}$. These interactions are the dominant ones at the early stages of preheating, when the amplitude of the inflaton is large. (We do not consider here nonrenormalizable interactions, which - if present-could be more important.) However, they do not lead to a complete inflaton decay. A complete decay requires vertices with only one inflaton, as the Yukawa interaction $\phi \bar{\psi} \psi$ with fermions, or the three-legs bosonic interaction $\sigma \phi \chi^{2}$ (where $\sigma$ is a mass scale). As we have argued, these trilinear interactions are very natural in supersymmetric models. Supersymmetry has the additional advantage of protecting the flatness of the inflaton potential from too large radiative corrections, which arise from the coupling of the inflaton to the light degrees of freedom.

(iv) Finally, the early evolution of the light degrees of freedom from the decay of the inflaton can have very important consequences for the generation of particles far from thermal equilibrium. A particularly relevant application is the possible (over)production of gravitinos from the decay products of the inflaton. Although this is a perturbative generation, it can be very effective, since it occurs at very early times. Traditionally, the limit $T_{r} \lesssim 10^{9} \mathrm{GeV}$ is due to gravitinos produced only after the $\chi$ quanta have thermalized. However, a nonthermal distribution can also be responsible for gravitino overproduction [28]. These inflaton decay products arise very rapidly, with an energy density much higher than $\left(10^{9} \mathrm{GeV}\right)^{4}$; although their initial distribution is far from thermal, we have seen that they can be responsible for a significant gravitino production, which in some cases exceeds by several orders magnitude the allowed bound. Clearly, this is a less universal bound than the standard one, since it depends on the details of how the thermal bath is produced. (For instance, the gravitino abundance can be diluted by entropy release during thermalization.) In addition, the gravitino problem is avoided altogether if the gravitino mass is significantly smaller (as in gauge mediated supersymmetry breaking) or larger (as in the simplest versions of the KKLT model [31]) than the electroweak scale. Still, this possible production must be addressed in many inflationary models.

\section{ACKNOWLEDGMENTS}

We thank J. Berges, J.F. Dufaux, A. Linde, A. Mazumdar, and I. Tkachev for useful discussions. G. F. and M. P. thank CITA for its warm hospitality during different stages of this work. L. K. was supported by CIAR and NSERC. M. P. was supported in part by the DOE Grant No. DE-FG02-94ER40823. 
[1] A. D. Dolgov and A.D. Linde, Phys. Lett. 116B, 329 (1982); L. F. Abbott, E. Fahri, and M. Wise, Phys. Lett. 117B, 29 (1982).

[2] R. Allahverdi, Phys. Rev. D 62, 063509 (2000); S. Davidson and S. Sarkar, J. High Energy Phys. 11 (2000) 012; R. Allahverdi and M. Drees, Phys. Rev. D 66, 063513 (2002).

[3] G. F. Giudice, E. W. Kolb, and A. Riotto, Phys. Rev. D 64, 023508 (2001).

[4] L. Kofman, A. D. Linde, and A. A. Starobinsky, Phys. Rev. Lett. 73, 3195 (1994).

[5] J. Traschen and R. Brandenberger, Phys. Rev. D 42, 2491 (1990).

[6] L. Kofman, A. D. Linde, and A. A. Starobinsky, Phys. Rev. D 56, 3258 (1997).

[7] G. N. Felder, J. Garcia-Bellido, P. B. Greene, L. Kofman, A. D. Linde, and I. Tkachev, Phys. Rev. Lett. 87, 011601 (2001); G. N. Felder, L. Kofman, and A. D. Linde, Phys. Rev. D 64, 123517 (2001).

[8] L. Kofman, astro-ph/0303614.

[9] G. Dvali, A. Gruzinov, and M. Zaldarriaga, Phys. Rev. D 69, 023505 (2004).

[10] F. Bernardeau, L. Kofman and J. P. Uzan, Phys. Rev. D 70, 083004 (2004).

[11] S. Y. Khlebnikov and I.I. Tkachev, Phys. Rev. Lett. 77, 219 (1996).

[12] T. Prokopec and T. G. Roos, Phys. Rev. D 55, 3768 (1997).

[13] G. N. Felder and L. Kofman, Phys. Rev. D 63, 103503 (2001).

[14] R. Micha and I. I. Tkachev, Phys. Rev. D 70, 043538 (2004); R. Micha and I. I. Tkachev, Phys. Rev. Lett. 90, 121301 (2003).

[15] G. N. Felder and I. Tkachev, hep-ph/0011159.

[16] D. Boyanovsky, D. Cormier, H. J. de Vega, R. Holman, A. Singh, and M. Srednicki, Phys. Rev. D 56, 1939 (1997).

[17] J. Berges and J. Serreau, hep-ph/0302210; J. Berges and J. Serreau, hep-ph/0410330.

[18] J. Berges, S. Borsanyi, and C. Wetterich, Phys. Rev. Lett. 93, 142002 (2004).

[19] R. Kallosh, L. Kofman, A. D. Linde, and A. Van Proeyen, Phys. Rev. D 61, 103503 (2000); Classical Quantum Gravity 17, 4269 (2000); G. F. Giudice, A. Riotto, and I. Tkachev, J. High Energy Phys. 08 (1999) 009 J. High Energy Phys. 11 (1999) 036; H. P. Nilles, M. Peloso, and
L. Sorbo, Phys. Rev. Lett. 87, 051302 (2001J. High Energy Phys. 04 (2001) 004.

[20] J. Ellis, A. Linde, and D. Nanopoulos, Phys. Lett. 118B, 59 (1982); L. M. Krauss, Nucl. Phys. B227, 556 (1983); D. Nanopoulos, K. Olive, and M. Srednicki, Phys. Lett. 127B, 30 (1983); M. Yu. Khlopov and A. Linde, Phys. Lett. 138B, 265 (1984); J. Ellis, J. Kim, and D. Nanopoulos, Phys. Lett. 145B, 181 (1984); J. Ellis, G. B. Gelmini, J. L. Lopez, D. V. Nanopoulos, and S. Sarkar, Nucl. Phys. B373, 399 (1992); M. Kawasaki and T. Moroi, Prog. Theor. Phys. 93, 879 (1995).

[21] J.F. Dufaux, G. Felder, L. Kofman, M. Peloso, and D. Podolsky, to be published.

[22] L. A. Kofman and A. D. Linde, Nucl. Phys. B282, 555 (1987).

[23] D. V. Semikoz and I. I. Tkachev, Phys. Rev. Lett. 74, 3093 (1995).

[24] J. Baacke, K. Heitmann, and C. Patzold, Phys. Rev. D 58, 125013 (1998); P. B. Greene and L. Kofman, Phys. Lett. B 448, 6 (1999); G. F. Giudice, M. Peloso, A. Riotto, and I. Tkachev, J. High Energy Phys. 08 (1999) 014; P. B. Greene and L. Kofman, Phys. Rev. D 62, 123516 (2000); M. Peloso and L. Sorbo, J. High Energy Phys. 05 (2000) 016.

[25] A. R. Liddle and D. H. Lyth, Cosmological Inflation and Large Scale Structure (Cambridge University Press, Cambridge, 2000).

[26] A. R. Liddle and D. H. Lyth, Phys. Rep. 231, 1 (1993).

[27] M. Kawasaki, K. Kohri, and T. Moroi, Phys. Lett. B 625, 7 (2005).

[28] L. Boubekeur, S. Davidson, M. Peloso, and L. Sorbo, Phys. Rev. D 67, 043515 (2003).

[29] For the entropy density we used the formula $s=$ $\int d^{3} k\left[\left(n_{k}+1\right) \ln \left(\mathrm{n}_{\mathrm{k}}+1\right)-\mathrm{n}_{\mathrm{k}} \ln \left(\mathrm{n}_{\mathrm{k}}\right)\right]$. See for example R.H. Brandenberger, T. Prokopec, and V. Mukhanov, Phys. Rev. D 48, 2443 (1993).

[30] D. H. Lyth and E. D. Stewart, Phys. Rev. Lett. 75, 201 (1995); L. Kofman, A. D. Linde, and A. A. Starobinsky, Phys. Rev. Lett. 76, 1011 (1996); G. N. Felder, L. Kofman, A. D. Linde, and I. Tkachev, J. High Energy Phys. 08 (2000) 010.

[31] R. Kallosh and A. Linde, J. High Energy Phys. 12 (2004) 004. 